\title{
Mini-Open Anterior Retroperitoneal Lumbar Interbody Fusion: Oblique Lateral Interbody Fusion for Degenerated Lumbar Spinal Kyphoscoliosis
}

Seiji Ohtori*, Chikato Mannoji*, Sumihisa Orita*, Kazuyo Yamauchi, Yawara Eguchi, Nobuyasu Ochiai, Shunji Kishida, Kazuki Kuniyoshi, Yasuchika Aoki, Junichi Nakamura,

Tetsuhiro Ishikawa, Masayuki Miyagi, Hiroto Kamoda, Miyako Suzuki, Gou Kubota, Yoshihiro Sakuma, Yasuhiro Oikawa, Kazuhide Inage, Takeshi Sainoh, Jun Sato, Yasuhiro Shiga, Koki Abe, Kazuki Fujimoto, Hiroto Kanamoto, Tomoaki Toyone, Gen Inoue, Kazuhisa Takahashi

Department of Orthopaedic Surgery, Graduate School of Medicine, Chiba University, Chiba, Japan

\section{Study Design: Prospective case series.}

Purpose: To examine the clinical efficacy of mini-open anterior retroperitoneal lumbar interbody fusion: oblique lateral interbody fusion (OLIF) for degenerated lumbar spinal kyphoscoliosis.

Overview of Literature: The existing surgical procedures for the treatment of spinal kyphotic deformity, including Smith-Petersen osteotomy, pedicle subtraction osteotomy, and vertebral column resection procedures, are invasive in nature. Extreme lateral interbody fusion to provide less invasive treatment of the deformity has been reported, but complications including spinal nerve and psoas muscle injury have been noted. In the current study, we examined the clinical efficacy and complications of OLIF for degenerated lumbar spinal kyphoscoliosis.

Methods: Twelve patients with degenerated lumbar spinal kyphoscoliosis were examined. All patients underwent OLIF surgery (using a cage and bone graft from the iliac crest) with open pedicle screws or percutaneous pedicle screws, without real-time monitoring by electromyography. Visual analog scale score and Oswestry disability index were evaluated before and 12 months after surgery, and fusion rate at OLIF cage, correction of the deformity, total blood loss, and surgical complications were also evaluated.

Results: Pain scores significantly improved after surgery $(p<0.05)$. Fusion rate was found to be $90 \%$, balance parameters also improved after surgery $(p<0.05)$, and average total blood loss was less than $350 \mathrm{~mL}$. There was no spinal nerve, major vessel, peritoneal, or urinary injury, or breakage of instrumentation.

Conclusions: OLIF surgery for degenerated lumbar spinal kyphoscoliosis is less invasive than other procedures and good surgical results were produced without major complications.

Keywords: Lumbar; Kyphoscoliosis; Nerve; Injury; Surgery

Received Nov 12, 2014; Revised Dec 9, 2014; Accepted Dec 10, 2014

Corresponding author: Seiji Ohtori

Department of Orthopaedic Surgery, Graduate School of Medicine, Chiba University, 1-8-1 Inohana, Chuo-ku, Chiba 260-8670, Japan Tel: +81-43-226-2117, Fax: +81-43-226-2116, E-mail: sohtori@faculty.chiba-u.jp

*Seiji Ohtori, Chikato Mannoji, and Sumihisa Orita contributed equally to this work. 


\section{Introduction}

Spinal kyphosis in elderly people can sometimes cause serious problems. It occurs as a consequence of the loss of the physiological lordotic curve because of senile kyphosis, which is attributed to osteoporosis, disk degeneration, and impairment of back muscles [1,2]. The postural abnormality associated with kyphosis can lead to chronic lower back pain, and in later stages it can also disturb standing and gait by affecting balance [3].

There have been many surgical procedures described for the treatment of spinal kyphotic deformity, including Smith-Petersen osteotomy, pedicle subtraction osteotomy, and vertebral column resection. The technique used to correct spinal kyphotic deformity depends on variables such as the severity of the deformity, the flexibility of the deformity, and whether the kyphosis is more of a rounded, long sweeping kyphosis, or short and angular $[4,5]$.

The minimally invasive lateral transpsoas approach to the lumbar spine is also known as extreme lateral interbody fusion (XLIF), as first described in 2001 [6,7]. The advantages of XLIF include minimally invasive access to the lumbar spine, and less blood loss when compared to open surgery [6]. XLIF is a promising minimally invasive option for adult deformity. It can be used in combination with standard posterior techniques or with percutaneous pedicle fixation. The role of XLIF plus minimally invasive posterior fixation in obtaining satisfactory curve correction, while reducing the number of levels fused in lumbar scoliosis, can be advantageous over other traditional techniques [8].

However, in XLIF, access to the lumbar spine is via a lateral approach that passes through the psoas major muscle, which consequently leads to several complications. It has been reported that after XLIF surgery, 30\% of patients show paresthesias in the leg and $27 \%$ of patients show thigh pain after XLIF surgery, indicating nerve injury [9]. Furthermore, high rates (62.7\%) of transient anterior thigh symptoms are found despite real-time electromyography (EMG) monitoring [10].

As with XLIF, to avoid nerve injury, mini-open anterior retroperitoneal lumbar interbody fusion methods, such as oblique lumbar interbody fusion (OLIF) have been applied in a total of 179 patients [11]. Less nerve injury has been reported following this method using an anterior approach between the aorta and psoas, when compared to XLIF. In the current study, a banana-shaped polyetheretherketone cage (Boomerang, Medtronic Inc., Minneapolis, MN, USA) was used [11]. Subsequently, an OLIF system (OLIF25, Medtronic Inc.) and cage (Clydesdale Spinal System, Medtronic Inc.) have been developed, and procedures using the OLIF25 system and Clydesdale Spinal System allowed a larger interbody graft to be placed into the disk space for anterior column support and segmental sagittal alignment, while minimizing the nerve, muscle, and bony obstacles associated with traditional direct lateral approaches.

To the best of our knowledge, results and complications of this system for degenerated lumbar spinal kyphoscoliosis have not yet been reported. In the current study, we aimed to examine the clinical efficacy of OLIF for degenerated lumbar spinal kyphoscoliosis and to determine any complications that occur during surgery.

\section{Materials and Methods}

\section{Patients and surgery}

We evaluated 12 patients in Chiba University Hospital between June 2012 and February 2013. Patients were diagnosed with degenerated lumbar kyphoscoliosis on $\mathrm{X}$-ray and magnetic resonance imaging (MRI), myelography, and computed tomography (CT) after myelography. Patients who had previously undergone spinal surgery and who had both spinal stenosis and kyphoscoliosis were included in the present study. Patients with spinal tumor, infection, and acute vertebral fractures of the thoracic and lumbar spine were excluded from the investigation. Patients met criteria for surgery for their kyphoscoliosis if they showed severe low back pain, or had difficulty in standing or walking after undergoing more than six months of conservative treatment. For diagnosis of degenerated kyphoscoliosis and indication for surgery, a Cobb angle of more than $40^{\circ}$ on the coronal plane or less than $10^{\circ}$ of lordosis on the sagittal plane of the lumbar spine were used as inclusion criteria. Informed consent was obtained from all research participants. Bone mineral density in the lumbar spine was examined before surgery. Details of the patients' backgrounds are shown in Table 1.

All patients initially underwent OLIF (OLIF25) surgery. A cage (Clydesdale Spinal System) filled with bone graft from the iliac bone was used in this study (Figs. 1, 2). Subsequently, posterior fixation was employed in all patients. Open pedicle screws or percutaneous pedicle 
Table 1. Demographic characteristics

\begin{tabular}{|c|c|}
\hline Characteristic & Value \\
\hline No. of patients & 12 \\
\hline \multicolumn{2}{|l|}{ Sex } \\
\hline Male & 4 \\
\hline Female & 8 \\
\hline Age (yr) & $64 \pm 4.5(58-78)$ \\
\hline Symptom duration (yr) & $10.0(6-20)$ \\
\hline Follow-up after surgery (mo) & $14.5(12-24)$ \\
\hline BMD (young adult mean) (\%) & $80 \pm 10.0(65-95)$ \\
\hline \multicolumn{2}{|l|}{ Complication before surgery } \\
\hline Diabetes mellitus & 2 \\
\hline Parkinson disease & 3 \\
\hline Revision surgery & 2 \\
\hline Hemodialysis & 1 \\
\hline Smoker & 1 \\
\hline \multicolumn{2}{|l|}{ No. of fusion levels (OLIF) } \\
\hline 2 & 3 \\
\hline 3 & 7 \\
\hline 4 & 2 \\
\hline \multicolumn{2}{|l|}{ No. of fusion levels (posterior) } \\
\hline 3 & 1 \\
\hline 4 & 4 \\
\hline 6 & 3 \\
\hline 9 & 1 \\
\hline 11 & 1 \\
\hline 15 & 2 \\
\hline \multicolumn{2}{|l|}{ Posterior fixation } \\
\hline Open PS & 4 \\
\hline PPS & 8 \\
\hline \multicolumn{2}{|l|}{ Posterior decompression } \\
\hline Performed & 1 \\
\hline Not performed & 11 \\
\hline
\end{tabular}

Values are presented as number or mean \pm standard deviation. BMD, bone mineral density; OLIF, oblique lateral interbody fusion; PS, pedicle screws; PPS, percutaneous pedicle screws.

screws were used in all patients. Hooks and iliac screws were used in some patients. Open pedicle screws or percutaneous pedicle screws were used in all patients. Open pedicle screws were used for longer fusion, and percutaneous pedicle screws were used for relatively shorter fusion. Posterior osteotomy or posterior bone grafting was not performed in any patient. Rods were bent along the

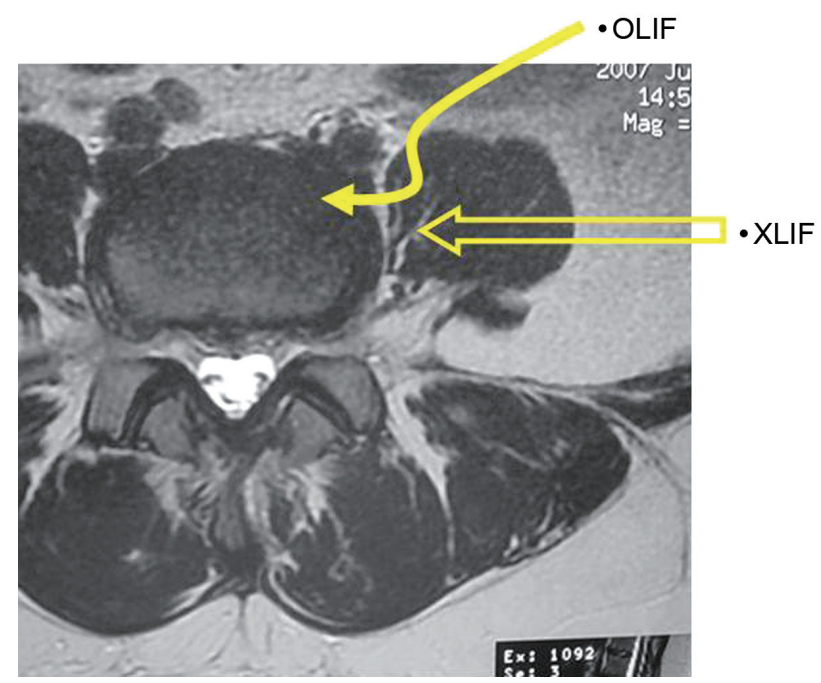

Fig. 1. Approach to oblique lateral interbody fusion (OLIF) and extreme lateral interbody fusion (XLIF). OLIF is an anterior to psoas approach.

heads of the pedicle screws, and inserted into the heads of pedicle screws using reducers. Strong sagittal or rotational reduction was not performed in any patient. OLIF fusion from 1 to 4 levels and posterior fusion from 3 to 15 levels were performed. Posterior decompression was performed in one patient, but not in the others.

\section{Radiographic and clinical evaluation}

Radiography was used to evaluate spinal variables before and after surgery. Anteroposterior and profile X-ray image views before and after surgery were examined. Measurements were made on radiographs obtained before surgery, and 12 months after surgery. Sagittal balance was assessed at a standing position, and sagittal vertical axis (SVA) was measured as the horizontal distance between a C7 plumb line and the posterosuperior aspect of the sacrum at the L5-S1 disk. Thoracic kyphosis was measured from T5T12 using the Cobb method. Lumbar lordosis (LL) was measured from T12 to the sacrum in a similar fashion. Coronal balance was measured at the maximum angle by the Cobb method [12].

Sacral slope was defined as the angle subtended by a horizontal reference line and a line drawn parallel to the sacral end plate. Pelvic incidence (PI) was defined as the angle subtended by a line dropped perpendicular to the sacral end plate and a line drawn from the center of the sacral end plate to the center of the bicoxofemoral axis. Pelvic tilt (PT) was defined as the angle subtended by a vertical 

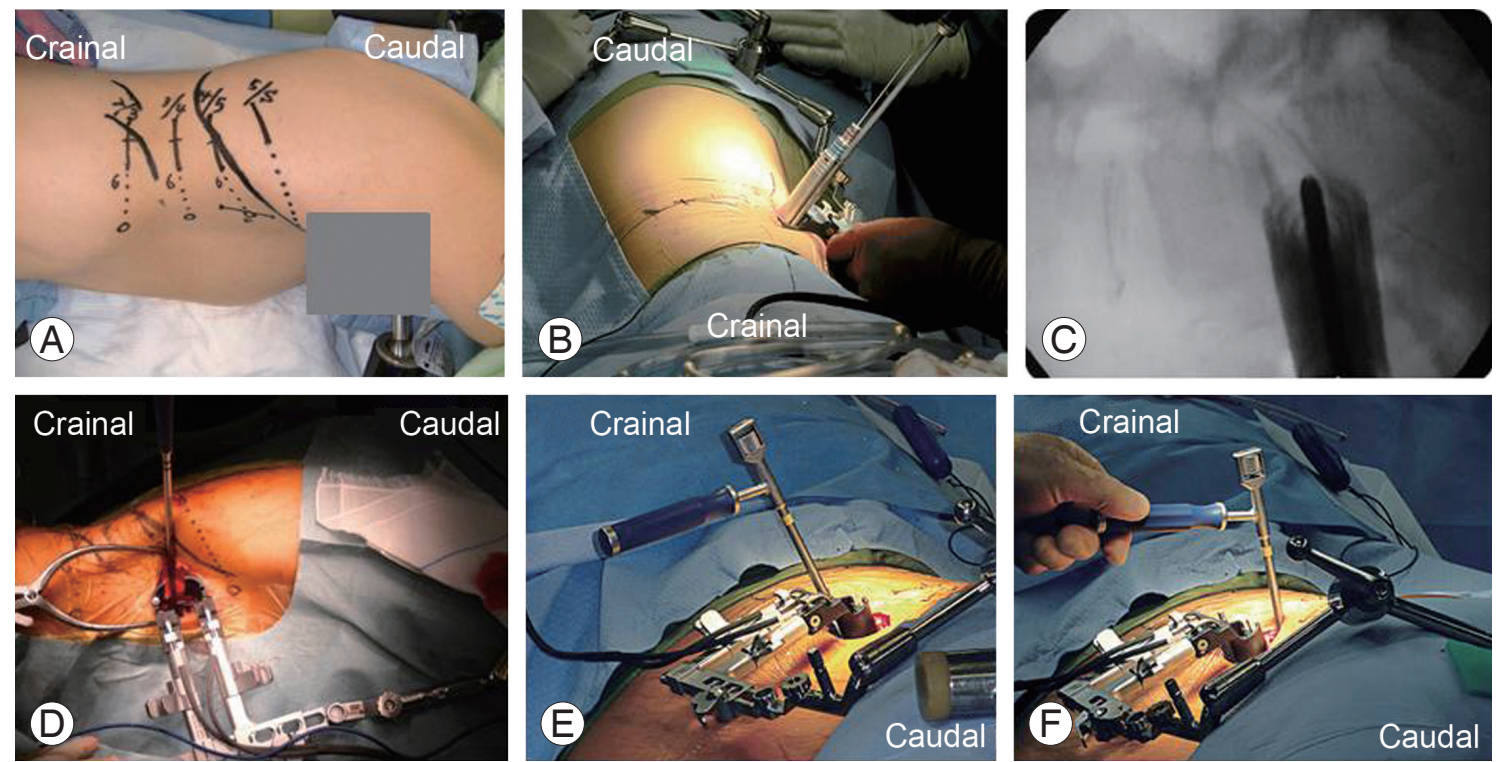

Fig. 2. (A) Skin marking to check the disk level using a C-arm X-ray imager. The skin incision was made 6 to $10 \mathrm{~cm}$ anterior to the midportion of the disk. A longitudinal incision from 3 to $4 \mathrm{~cm}$ is recommended. The surgeon cleared the peritoneum from the psoas using a finger. (B) Sequential dilation was used. (C) C-arm X-ray image after dilation. (D) Retractor for oblique lateral interbody fusion was used after dilatation. A trial cage was used after removal of the intervertebral disk. (E, F) A Clydesdale Spinal System cage filled with autologous bone is implanted. Implantation starts from a lateral oblique direction; finally the cage is inserted from a true lateral direction.

reference line and a line drawn from the center of the sacral end plate to the center of the bicoxofemoral axis [12].

CT was performed to evaluate bone union 12 months after surgery. Here we defined bone union as bridging bone formation between adjacent vertebral bodies (at the OLIF cage). Evaluation of bone union was blinded and performed by 3 surgeons. Fusion was identified if at least 2 of the observers concurred.

The change in lower back pain before and after surgery was evaluated using a visual analogue scale score $(0$, no pain; 10, worst pain). The Oswestry disability index for low back pain was recorded before, and 1 and 12 months after surgery, and the findings were compared.

\section{Surgical time, blood loss, and complications}

Surgical time, blood loss, and complications during and after surgery were investigated.

\section{Statistical analyses}

To determine whether there were significant changes in pain score and change in spinal variables after surgery, $t$-test and chi-square test were used. A $p<0.05$ was consid- ered significant.

\section{Results}

Table 1 shows demographic characteristics of the patients before surgery. Four men and eight women were included in this study. Concurrent conditions included diabetes mellitus in two patients, Parkinson disease in two patients, revision surgery (prior posterior surgery) in two patients, and hemodialysis in two patients. One patient was a smoker. The OLIF procedure was performed from 2 to 4 levels for 35 levels in 12 patients. Open pedicle screws (four patients) and percutaneous pedicle screws (eight patients) were used for posterior fixation. Posterior fusion was performed from 3 to 15 levels.

\section{Change of spinal variables}

SVA and Cobb angle of the coronal plane and LL were significantly improved at the final follow-up compared with the values before surgery $(p<0.05)$ (Table 2 , Figs. 3, 4). SVA was $27 \mathrm{~mm}$ (less than $50 \mathrm{~mm}$ ); PT was $23^{\circ}$ (less than $25^{\circ}$ ); LL was $37^{\circ}$; and PI was $45^{\circ}$ after surgery. The difference between LL and PI was $8^{\circ}$ after surgery. 
Table 2. Average change of spinal parameter

\begin{tabular}{|c|c|c|c|c|c|}
\hline \multirow{2}{*}{ Variable } & \multicolumn{2}{|c|}{ Before surgery } & \multicolumn{2}{|c|}{ After surgery } & \multirow{2}{*}{$p$-value } \\
\hline & Mean & $\mathrm{SD}$ & Mean & $\mathrm{SD}$ & \\
\hline Sagittal vertical axis (mm) & 140 & 20 & 27 & 6 & 0.005 \\
\hline Coronal (cobb) $\left({ }^{\circ}\right)$ & 42 & 7 & 5 & 2 & 0.010 \\
\hline Lumbar lordosis $\left({ }^{\circ}\right)$ & 6 & 1 & 37 & 10 & 0.020 \\
\hline Thoracic kyphosis ( $\left.{ }^{\circ}\right)$ & 15 & 3 & 24 & 8 & 0.045 \\
\hline Pelvic tilt $\left({ }^{\circ}\right)$ & 37 & 7 & 23 & 7 & 0.040 \\
\hline Sacral slope $\left({ }^{\circ}\right)$ & 10 & 3 & 22 & 4 & 0.033 \\
\hline Pelvic incidence $\left({ }^{\circ}\right)$ & 47 & 10 & 45 & 11 & 0.230 \\
\hline
\end{tabular}

SD, standard deviation.
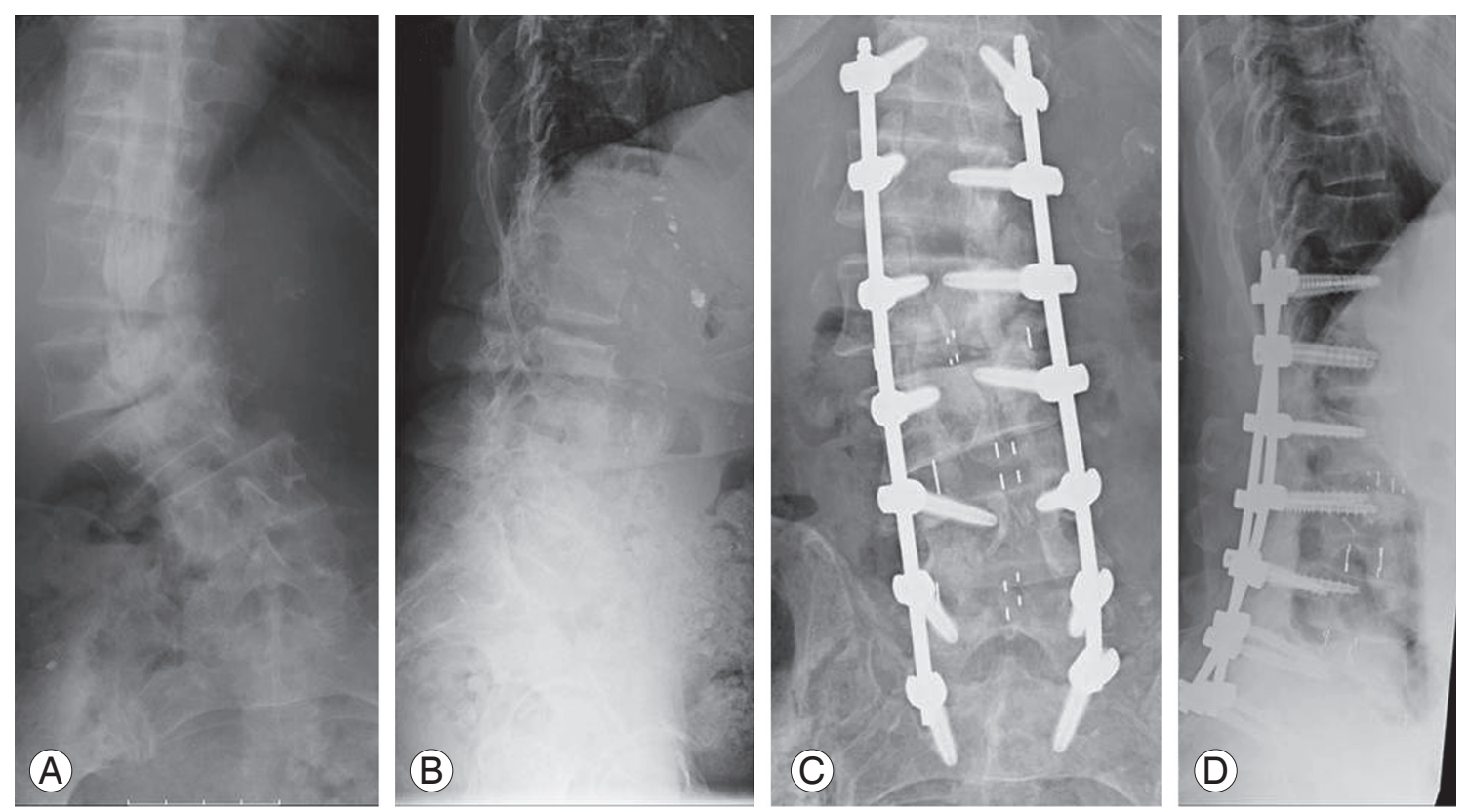

Fig. 3. A 68-year-old woman with hemodialysis over 30 years showing kyphoscoliosis. X-ray images showing severe kyphoscoliosis. (A, B) Improvement in kyphoscoliosis, (C, D) after surgery (3-level oblique lateral interbody fusion and open pedicle screws with posterior decompression).

1) Bone fusion

Fusion was seen at 31 of 35 levels, and the rate of fusion was about $90 \%$. Four levels were not fuse; however, the patients showed no problems and did not require revision surgery.

\section{2) Pain score}

Low back pain, as evaluated using a visual analogue scale score, was significantly improved from $9.5 \pm 3.5$ to $2.3 \pm 1.7$ $(p=0.005)$ and Oswestry disability index was significantly improved from $72 \pm 22$ to $22 \pm 12$ ( $p=0.033$ ).

\section{Surgical time, blood loss, and complications}

Average surgical time and average blood loss during surgery were observed to be $250 \pm 35$ minutes and $350 \pm 50$ $\mathrm{mL}$, respectively. OLIF appeared less invasive when compared to other procedures. There was no infection, major vessel injury, peritoneal injury, or urinary injury in patients. Hardware failure did not occur, however, cage subsidence at 1 level was observed in 1 patient with osteoporotic kyphoscoliosis. There was no occurrence of spinal nerve injury. Thigh pain was observed in one patient and 

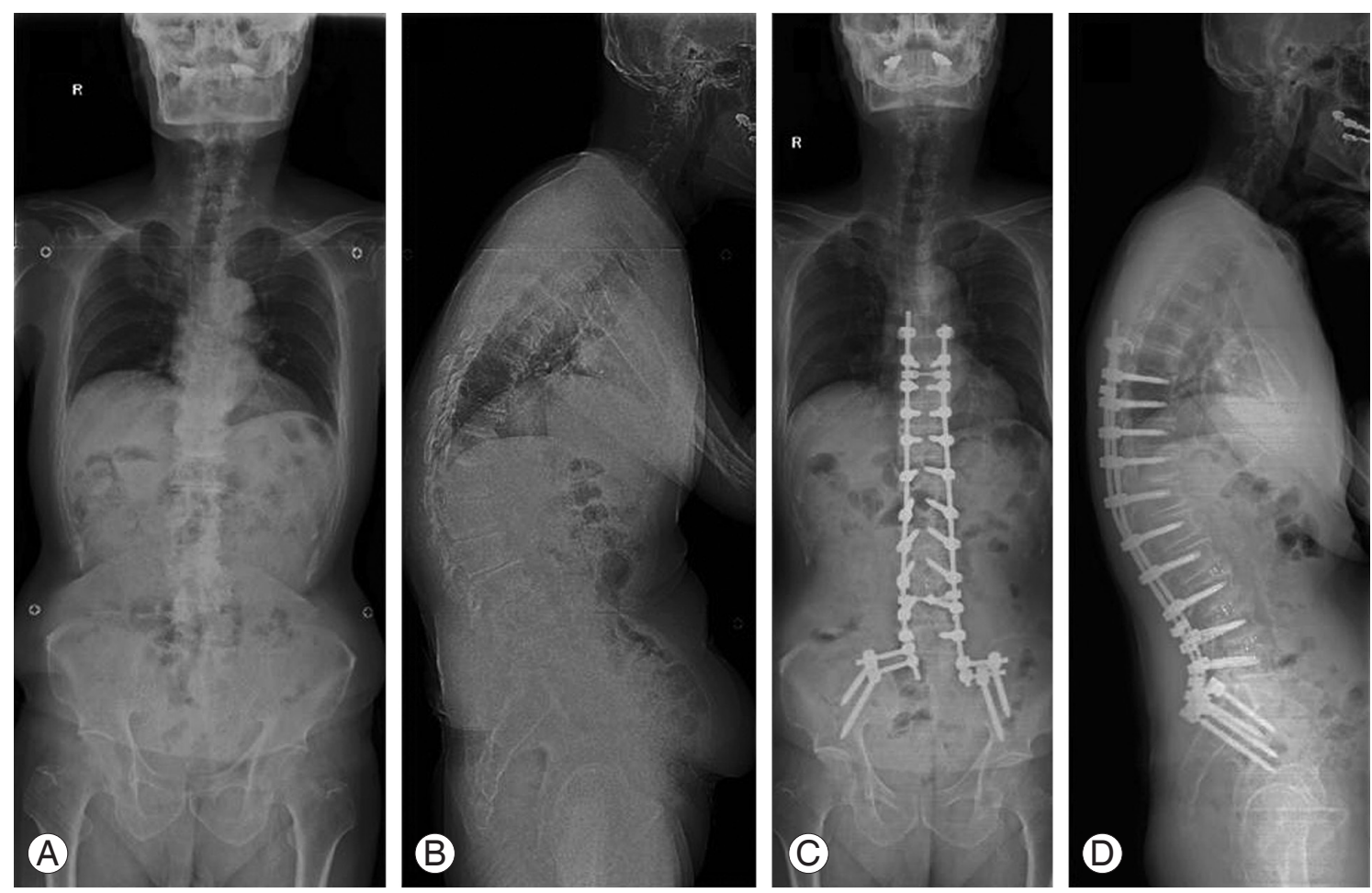

Fig. 4. A 66-year-old woman with kyphoscoliosis. (A, B) The entire spine before surgery and (C, D) 1 year after surgery is shown.

thigh numbness in two patients. Thigh pain and numbness diminished within two weeks of surgery. Donor site (iliac crest) pain was seen frequently and occurrence was noted in eight patients.

\section{Discussion}

In the current study, we evaluated OLIF for degenerated lumbar spinal kyphoscoliosis. There were few complications during surgery. In particular, injury to psoas muscles and spinal nerves was avoided. Fusion rate and correction of deformity were satisfactory and lower back pain decreased significantly after surgery.

Recently, Acosta et al. [13] analyzed changes in coronal and sagittal plane alignment following XLIF for degenerative scoliosis and noted excellent results for deformity correction in both planes. Other authors recently used a combined approach of minimally invasive XLIF and open posterior segmental pedicle screw instrumentation with transforaminal lumbar interbody fusion for the correction of coronal deformity. Radiographic outcomes such as the Cobb angle and apical vertebral translation were significantly improved and less blood loss was noted in patients; the radiographic outcomes were comparable to those of traditional posterior-only approaches [14]. Schwab et al. [12] reported that realignment objectives should be patient-specific and involve attention to the following 3 parameters: SVA less than $50 \mathrm{~mm}$, PT less than $25^{\circ}$, and $\mathrm{LL}=\mathrm{PI} \pm 9^{\circ}$ [12]. In the current study, spinal variables were within these parameters, therefore, we conclude that OLIF plus posterior fixation is sufficient to correct adult deformity.

For minimally invasive surgery, recent studies on surgical treatment of adult scoliosis have found that XLIF results in less blood loss, a shorter length of stay, and a lower incidence of infection when compared to traditional open procedures $[15,16]$. A prospective study of 107 patients, who underwent the XLIF procedure with or without supplemental percutaneous posterior fusion for the treatment of degenerative scoliosis found the mean operative time of 178 minutes and blood loss of between $50 \mathrm{~mL}$ and $100 \mathrm{~mL}$ [17]. Thirty consecutive patients were followed for an average of 14.3 months to determine fusion rate [18]. There was significant improvement in multiple radiographic variables postoperatively, and there was no significant loss of correction postoperatively up until the most recent follow-up. However, there was an $11.8 \%$ incidence of pseudoarthrosis at levels treated with XLIF 
[18]. The results of the current study are similar to those previously reported for XLIF.

Many authors have reported complications of XLIF during surgery. Symptoms included burning, aching, stabbing, or other pain, numbness, paresthesias, or weakness. Davis et al. [19] have reported the course of the lumbar plexus using cadaveric specimens. The femoral nerve lies ventral to its posterior aspect, and there is a possibility of injury to the femoral nerve during the approach to L4-5 using XLIF [19]. Furthermore, Uribe et al. [20] discussed the potential of injury to the ilioinguinal, iliohypogastric, lateral femoral cutaneous, and genitofemoral nerves in the retroperitoneal space because real-time EMG monitoring does not monitor sensory nerves. Especially, at the L4-5 level, the genitofemoral nerve, the ilioinguinal, and iliohypogastric nerves run from anterior to posterior of the disk [20]. In the current study, we approached the disk between the aorta and psoas muscle without real-time EMG monitoring, and few patients showed any motor or sensory nerve injury or symptoms from the psoas muscle. In this regard, the demonstrated OLIF method is useful for avoiding the complications reported for the XLIF procedure.

The current study has some limitations. It is a smallsized prospective study and the number of patients was restricted. The duration of follow-up was also short. Further follow-up is needed to strengthen these observations.

We conclude that OLIF plus posterior open or percutaneous pedicle screws is an option for correction of deformity due to degenerated lumbar spinal kyphoscoliosis.

\section{Conclusions}

OLIF surgery for degenerated lumbar spinal kyphoscoliosis is less invasive than other procedures and good surgical results were produced without any major complications.

\section{Conflict of Interest}

No potential conflict of interest relevant to this article was reported.

\section{References}

1. Bartynski WS, Heller MT, Grahovac SZ, Rothfus WE, Kurs-Lasky M. Severe thoracic kyphosis in the older patient in the absence of vertebral fracture: association of extreme curve with age. AJNR Am J Neuroradiol 2005;26:2077-85.

2. Takemitsu Y, Harada Y, Iwahara T, Miyamoto M, Miyatake Y. Lumbar degenerative kyphosis: clinical, radiological and epidemiological studies. Spine (Phila Pa 1976) 1988;13:1317-26.

3. Booth KC, Bridwell KH, Lenke LG, Baldus CR, Blanke KM. Complications and predictive factors for the successful treatment of flatback deformity (fixed sagittal imbalance). Spine (Phila Pa 1976) 1999;24:171220.

4. Bridwell KH. Decision making regarding Smith-Petersen vs. pedicle subtraction osteotomy vs. vertebral column resection for spinal deformity. Spine (Phila Pa 1976) 2006;31(19 Suppl):S171-8.

5. Suk SI, Chung ER, Kim JH, Kim SS, Lee JS, Choi WK. Posterior vertebral column resection for severe rigid scoliosis. Spine (Phila Pa 1976) 2005;30:1682-7.

6. Ozgur BM, Aryan HE, Pimenta L, Taylor WR. Extreme lateral interbody fusion (XLIF): a novel surgical technique for anterior lumbar interbody fusion. Spine J 2006;6:435-43.

7. Pimenta L. Lateral endoscopic transpsoas retroperitoneal approach for lumbar spine surgery. In: Proceedings of the VIII Brazilian Spine Society Meeting; 2001 May; Minas Gerais, Brazil.

8. Berjano P, Lamartina C. Far lateral approaches (XLIF) in adult scoliosis. Eur Spine J 2013;22 Suppl 2:S24253.

9. Bergey DL, Villavicencio AT, Goldstein T, Regan JJ. Endoscopic lateral transpsoas approach to the lumbar spine. Spine (Phila Pa 1976) 2004;29:1681-8.

10. Cummock MD, Vanni S, Levi AD, Yu Y, Wang MY. An analysis of postoperative thigh symptoms after minimally invasive transpsoas lumbar interbody fusion. J Neurosurg Spine 2011;15:11-8.

11. Silvestre C, Mac-Thiong JM, Hilmi R, Roussouly P. Complications and morbidities of mini-open anterior retroperitoneal lumbar interbody fusion: oblique lumbar interbody fusion in 179 patients. Asian Spine J 2012;6:89-97.

12. Schwab F, Patel A, Ungar B, Farcy JP, Lafage V. Adult spinal deformity-postoperative standing imbalance: how much can you tolerate? An overview of key parameters in assessing alignment and planning corrective surgery. Spine (Phila Pa 1976) 2010;35:2224-31. 
13. Acosta FL, Liu J, Slimack N, Moller D, Fessler R, Kos$\mathrm{ki}$ T. Changes in coronal and sagittal plane alignment following minimally invasive direct lateral interbody fusion for the treatment of degenerative lumbar disease in adults: a radiographic study. J Neurosurg Spine 2011;15:92-6.

14. Tormenti MJ, Maserati MB, Bonfield CM, Okonkwo DO, Kanter AS. Complications and radiographic correction in adult scoliosis following combined transpsoas extreme lateral interbody fusion and posterior pedicle screw instrumentation. Neurosurg Focus 2010;28:E7.

15. Anand N, Baron EM, Thaiyananthan G, Khalsa K, Goldstein TB. Minimally invasive multilevel percutaneous correction and fusion for adult lumbar degenerative scoliosis: a technique and feasibility study. J Spinal Disord Tech 2008;21:459-67.

16. Anand N, Rosemann R, Khalsa B, Baron EM. Midterm to long-term clinical and functional outcomes of minimally invasive correction and fusion for adults with scoliosis. Neurosurg Focus 2010;28:E6.

17. Isaacs RE, Hyde J, Goodrich JA, Rodgers WB, Phillips FM. A prospective, nonrandomized, multicenter evaluation of extreme lateral interbody fusion for the treatment of adult degenerative scoliosis: perioperative outcomes and complications. Spine (Phila Pa 1976) 2010;35(26 Suppl):S322-30.

18. Caputo AM, Michael KW, Chapman TM, et al. Extreme lateral interbody fusion for the treatment of adult degenerative scoliosis. J Clin Neurosci 2013;20: 1558-63.

19. Davis TT, Bae HW, Mok JM, Rasouli A, Delamarter RB. Lumbar plexus anatomy within the psoas muscle: implications for the transpsoas lateral approach to the L4-L5 disc. J Bone Joint Surg Am 2011;93:1482-7.

20. Uribe JS, Arredondo N, Dakwar E, Vale FL. Defining the safe working zones using the minimally invasive lateral retroperitoneal transpsoas approach: an anatomical study. J Neurosurg Spine 2010;13:260-6. 